\title{
Characterization of Human Prostate and Breast Cancer Cell Lines for Experimental TCell-Based Immunotherapy
}

\author{
Björn Carlsson, Ole Forsberg, Mats Bengtsson, Thomas H. Tötterman, \\ and Magnus Essand* \\ Clinical Immunology, Rudbeck Laboratory, Uppsala University, Uppsala, Sweden
}

\begin{abstract}
BACKGROUND. In order to develop experimental immunotherapy for prostate and breast cancer it is of outmost importance to have representative target cell lines that through human leukocyte antigen (HLA) class I molecules present relevant levels of peptides from tumorassociated antigens for cytotoxic T lymphocyte (CTL) recognition.

METHODS. We sequenced the HLA-A and HLA-B loci of eight commonly used prostate and breast cancer cell lines and analyzed the surface expression of HLA-ABC, HLA-DR, CD40, CD80, CD86, and CD54 by flow cytometry. We also analyzed the cell lines for mRNA expression from 25 genes reported to be specifically or preferentially expressed by prostate cells.

RESULTS. Among the analyzed cell lines we found that LNCaP, PC-346C and MCF-7 are HLA-A*0201 positive. However, the HLA-A2 expression level is low and only MCF-7 upregulates HLA-A2 in response to IFN- $\gamma$ stimulation. MCF-7 also expresses high levels of CD54, which further improve its value as a CTL target cell line. On the other hand, LNCaP and PC-346C express 25 and 23 out of 25 prostate-related genes, respectively, while MCF-7 expresses 16 out of 25 genes.

CONCLUSIONS. None of the analyzed prostate cancer cell lines are optimal CTL target cells. However, MCF-7 could in many cases be used as a complement to HLA-A*0201 positive prostate cancer cells. The LNCaP and PC-346C cell lines are rich sources of prostate-related antigens that may be valuable for cancer vaccine development. Prostate 67: 389-395, 2007.

(C) 2007 Wiley-Liss, Inc.
\end{abstract}

KEY WORDS: cell line; tumor-associated antigen; HLA; immunotherapy

\section{INTRODUCTION}

There are, today, no curative therapies for locally advanced or recurrent metastatic prostate cancer and novel treatment options are urgently needed. Immunotherapy with the aim to activate tumor-associated antigen (TAA)-reactive $\mathrm{T}$ cells is particularly well suited for prostate cancer. An important reason is that a large number of proteins with specific or preferential expression in normal prostate and prostate cancer cells have been identified [1]. Since the prostate gland is an organ that one can live without, these proteins constitute suitable targets for T cell-based immunotherapy. Immune responses to prostate-related antigens can be considered tumor-specific in patients with recurrent metastases after radical prostatectomy. Furthermore, serum prostate specific antigen (PSA) can be used as a surrogate marker to evaluate clinical responses. Breast cancer share many features with prostate cancer and strategies for prostate cancer immunotherapy can in many cases be applied also for breast cancer. For example, PSA and other kallikreins that are expressed by prostate cancer cells are also found in breast cancer cells $[2,3]$.

Grant sponsor: The Swedish Cancer Society; Grant number: 4419B05-06XBC; Grant sponsor: Swedish Research Council; Grant number: K2005-31X-15270-01A.

*Correspondence to: Magnus Essand, Clinical Immunology, Rudbeck Laboratory, Uppsala University, Uppsala, Sweden.

E-mail: magnus.essand@klinimm.uu.se

Received 22 May 2006; Accepted 5 July 2006

DOI 10.1002 / pros.20498

Published online 11 January 2007 in Wiley InterScience (www.interscience.wiley.com). 
The major cell type that mediates the rejection of solid tumors in preclinical animal models is the cytotoxic T lymphocyte (CTL). CTLs recognize 9-11 amino acid long peptide fragments bound to major histocompatibility complex (MHC) class I molecules, also known as human leukocyte antigen (HLA)-ABC, on the surface of the antigen presenting cell or target cell. Successful experimental cancer immunotherapy requires representative cell lines that can be used as target cells to verify the efficacy of activated CTLs. It is therefore of particular interest to know the HLA-ABC genotype and HLA-ABC expression level of the target cell lines. Cancer cell lines can also be used as antigen presenting cells for $\mathrm{T}$ cell activation, that is, as cancer vaccines. It is then of importance to know the expression levels of costimulatory molecules such as B7.1 (CD80), B7.2 (CD86), and CD40 as well as adhesion molecules such as ICAM-1 (CD54) since these molecules highly influence the quality of $\mathrm{T}$ cell activation. Furthermore, it is of outmost importance to know whether the laboratory cell lines express TAAs found in clinical cancer. Many of these characteristics have not yet been established and we therefore set out to sequence the HLA-A and HLA-B loci of six commonly used prostate cancer and immortalized normal prostate epithelial cell lines and two breast cancer cell lines. We also analyzed their surface expression of HLA-ABC (MHC class I), HLA-DR (MHC class II), CD40, CD80, CD86, and CD54 and investigated how the expression is influenced by exposure to interferon gamma (IFN- $\gamma$ ). In addition, we have analyzed the cell lines for mRNA expression of a large number of genes reported to be specifically or preferentially expressed by normal prostate and prostate cancer cells.

\section{MATERIALS AND METHODS}

\section{Cell Lines}

The human prostate adenocarcinoma cell lines LNCaP [4], PC-3 [5] and DU145 [6], and the immortalized normal human prostate epithelial cell lines BPH-1 [7] were obtained from ATCC (Rockville, MD). The prostate adenocarcinoma cell line PC-346C [8] was obtained from Dr. C Bangma, Erasmus University, Rotterdam, The Netherlands. The immortalized normal human prostate epithelial cell lines PNT2-C2 [9] and the human breast carcinoma cell line T47D [10] were obtained from Dr. N Maitland, York University, York, UK. The human breast carcinoma cell line MCF-7 [11] was obtained from Dr. J Berg, Karolinska Institute, Stockholm, Sweden. LNCaP, PC-346C, PC-3, DU145, BPH-1, PNT2-C2, and MCF-7 were cultured in RPMI1640 supplemented with $10 \%$ fetal bovine serum (FBS), $2 \mathrm{mM}$ L-glutamine, $10 \mathrm{mM}$ HEPES and $1 \mathrm{mM}$ sodium pyruvate while T47D was cultured in DMEM supple- mented with 5\% FBS and 2 mM L-glutamine. All cell culture reagents were from Invitrogen (Carlsbad, CA).

\section{Sequencing of the HLA-A and HLA-B Loci}

The HLA-A and HLA-B loci of the immortalized prostate, prostate cancer, and breast cancer cell lines were sequenced using previously described methods [12].

\section{Flow Cytometry Analysis}

Prostate and breast cancer cell lines were analyzed by flow cytometry (FACSCalibur ${ }^{\mathrm{TM}}$, BD Biosciences, San Jose, CA) using the following antibodies: FITClabeled anti-HLA-ABC (BD Biosciences), FITC-labeled anti-HLA-A2/A28 (One Lambda, Canoga Park, CA), PE-labeled anti-HLA-DR (BD Biosciences), FITClabeled anti-CD40 (BD Biosciences), PE-labeled antiCD80 (BD Biosciences), APC-labeled anti-CD86 (BD Biosciences), and APC-labeled anti-CD54 (BD Biosciences). A fraction of cells were pretreated with 1,000 IU/ml IFN- $\gamma$ (Imukin ${ }^{\mathrm{TM}}$, Boehringer-Ingelheim, Ingelheim, Germany) for $72 \mathrm{hr}$. Unspecific antibody bindings were analyzed by staining with isotypematch FITC-, APC-, and PE-labeled control antibodies (BD Biosciences).

\section{Reverse-Transcription (RT)-PCR}

To evaluate expression of 25 prostate-associated antigens, total RNA was isolated from all cell lines using TRIzol (Invitrogen) and cDNA was synthesized using Superscript II reverse transcriptase (Invitrogen) according to the manufacturer's instructions. Five micrograms of total RNA were used for each cDNA synthesis and an oligo-dT primer and a random hexamer primer were used in parallel reactions. Onetenth of the cDNA obtained from the oligo-dT cDNA synthesis and one-tenth of the cDNA obtained from the random hexamer cDNA synthesis were pooled and used as template for 35 cycles of PCR using the Expand $^{\mathrm{TM}}$ polymerase enzyme mix (Roche, Mannheim, Germany). In order to amplify cDNA only and not trace amounts of genomic DNA the primer pairs for each transcript were designed to generate PCR products spanning at least two exons. The primer sequences, annealing temperatures, and expected PCR product sizes are shown in Table I. PCR products were visualized by ethidium bromide staining on $2 \%$ agarose gels.

\section{RESULTS}

\section{HLA-A and HLA-B Loci Sequences of Prostate and Breast Cancer Cell Lines}

Prostate and breast cancer cell lines are often used as target cells for experimental immunotherapy and in 
TABLE I. Oligonucleotide Primers for RT-PCR Analyses of ProstateTissue Antigen Expression

\begin{tabular}{|c|c|c|c|}
\hline Target & Primers $\left(5^{\prime}\right.$ to $\left.3^{\prime}\right)$ & Annealing temperatures & Product size \\
\hline \multirow[t]{2}{*}{ PAP } & CATTGACACCTTTCCCACTG (F) & $51^{\circ} \mathrm{C}$ & $353 \mathrm{bp}$ \\
\hline & GTTCTTGAAAACGAGGGCAG (R) & & \\
\hline \multirow[t]{2}{*}{ PSA } & AGCCCCAAGCTTACCACC (F) & $53^{\circ} \mathrm{C}$ & $322 \mathrm{bp}$ \\
\hline & GCTGACCTGAAATACCTG(R) & & \\
\hline \multirow[t]{2}{*}{ hKLK4 } & GAACGGCAGAATGCCTACC (F) & $51^{\circ} \mathrm{C}$ & $294 \mathrm{bp}$ \\
\hline & TTAACTGGCCTGGACGGTTTTC (R) & & \\
\hline \multirow[t]{2}{*}{ PSP94 } & CACCATGAATGTTCTCCTGGGCAGCG (F) & $51^{\circ} \mathrm{C}$ & $347 \mathrm{bp}$ \\
\hline & TATCCATTCACTGACAGAACAGG (R) & & \\
\hline \multirow{2}{*}{ STAMP1 } & GCAGTCACTTCTATCCСTTC (F) & $51^{\circ} \mathrm{C}$ & $351 \mathrm{bp}$ \\
\hline & TCACATTACTGTGACCCTCTCCGG (R) & & \\
\hline \multirow{2}{*}{ Cten } & AGAGAGAGAACTGGGAGGTGC (F) & $51^{\circ} \mathrm{C}$ & $482 \mathrm{bp}$ \\
\hline & CTACATCCTTTCTGCGTCCTGC (R) & & \\
\hline \multirow[t]{2}{*}{ hKLK2 } & CGAACCAGAGGAGTTCTTGC (F) & $51^{\circ} \mathrm{C}$ & $307 \mathrm{bp}$ \\
\hline & TCAGGGGTTGGCTGCGATGGTG (R) & & \\
\hline \multirow[t]{2}{*}{ TARP } & GGGAACACCATGAAGACTAACGACAC (F) & $60^{\circ} \mathrm{C}$ & $339 \mathrm{bp}$ \\
\hline & TTTCTCTCCATTGCAGCAGAAAGCCG (R) & & \\
\hline \multirow[t]{2}{*}{ PDEF } & AAAGAGCGGACTTCACCTG (F) & $51^{\circ} \mathrm{C}$ & $373 \mathrm{bp}$ \\
\hline & TCAGATGGGGTGCACGAACTGG (R) & & \\
\hline \multirow[t]{2}{*}{ PSGR } & CACCATGAGTTCCTGCAACTTCACAC (F) & $51^{\circ} \mathrm{C}$ & $962 \mathrm{bp}$ \\
\hline & TCACTTGCCTCCCACAGCCTGC (R) & & \\
\hline \multirow{2}{*}{ PART1 } & CACCATGCAATGTCAGCTATTTAGGAC (F) & $51^{\circ} \mathrm{C}$ & $179 \mathrm{bp}$ \\
\hline & CTAAGTGATTGGCTGGCTCTGG (R) & & \\
\hline \multirow{2}{*}{ AIbZIP } & CAGACCAGCACTTGTGTTTTG (F) & $51^{\circ} \mathrm{C}$ & $312 \mathrm{bp}$ \\
\hline & TCACATCTCATCTGCATGCAGC (R) & & \\
\hline \multirow{2}{*}{ Trp-p8 } & CATTGTGTGTTTTGCCCAAG (F) & $51^{\circ} \mathrm{C}$ & $580 \mathrm{bp}$ \\
\hline & TGAGGACATCATGGGTGAGA (R) & & \\
\hline \multirow{2}{*}{ POTE } & CAAGTGGTGCTGTCACTGCT (F) & $51^{\circ} \mathrm{C}$ & $545 \mathrm{bp}$ \\
\hline & ATGTACGCCAAGCAAAAGTGGT (R) & & \\
\hline \multirow{2}{*}{ PMEPA1 } & CACCATGGCGGAGCTGGAGTTTGTTC (F) & $51^{\circ} \mathrm{C}$ & $758 \mathrm{bp}$ \\
\hline & CTAGAGAGGGTGTCCTTTCTGT (R) & & \\
\hline \multirow[t]{2}{*}{ PCTA1 } & CGTAGTGTTCTTTGGACACG (F) & $51^{\circ} \mathrm{C}$ & $420 \mathrm{bp}$ \\
\hline & CTACCAGCTCCTTACTTCCAGT (R) & & \\
\hline \multirow{2}{*}{ Prostein } & CCTTCACGCTGTTTTACACG (F) & $51^{\circ} \mathrm{C}$ & $799 \mathrm{bp}$ \\
\hline & CTACGCTGAGTATTTGGCCAAG (R) & & \\
\hline \multirow[t]{2}{*}{ PSMA } & CCAGGTTCGAGGAGGGATGGTG (F) & $51^{\circ} \mathrm{C}$ & $450 \mathrm{bp}$ \\
\hline & GCTACTTCACTCAAAGTATCTG (R) & & \\
\hline \multirow[t]{2}{*}{ PSDR1 } & CAACATCСТCTTCACCCAGG (F) & $51^{\circ} \mathrm{C}$ & $333 \mathrm{bp}$ \\
\hline & TTAGTCTATTGGGAGGCCCAGC (R) & & \\
\hline \multirow[t]{2}{*}{ TGM4 } & CATCATTGCGGAAATTGTGG (F) & $50^{\circ} \mathrm{C}$ & $364 \mathrm{bp}$ \\
\hline & CTACTTGGTTGATGAGAACAATC (R) & & \\
\hline \multirow[t]{2}{*}{ PSCA } & TGCTTGCCCTGTTGATGGCAG (F) & $53^{\circ} \mathrm{C}$ & $320 \mathrm{bp}$ \\
\hline & CCAGAGCAGCAGGCCGAGTGCA (R) & & \\
\hline STEAP & CACCATGGAAAGCAGAAAAGACATCA (F) & $53^{\circ} \mathrm{C}$ & $1,019 \mathrm{bp}$ \\
\hline & CTACAACTGGGAACATATCTCA (R) & & \\
\hline NGEP & ACAGCACCGTCCTGATCGATGTGAGC (F) & $60^{\circ} \mathrm{C}$ & $176 \mathrm{bp}$ \\
\hline & TGTCTAGCTTCAGGTCCTCСТСCCAA (R) & & \\
\hline PrLZ & GTAGAGAGATGGACTTATATGAGG (F) & $55^{\circ} \mathrm{C}$ & $668 \mathrm{bp}$ \\
\hline & TCACAGGCTCTCCTGTGTCTTTTC (R) & & \\
\hline NKX 3.1 & CAGAACGACCAGCTGAGCAC (F) & $53^{\circ} \mathrm{C}$ & $227 \mathrm{bp}$ \\
\hline & CGTTCAGGGGCCGACAGGTAC (R) & & \\
\hline$\beta$-actin & CTGGCACCCAGCACAATGAAGATCAA (F) & $51^{\circ} \mathrm{C}$ & $392 \mathrm{bp}$ \\
\hline & TCAAAGTCCTCGGCCACATTGTGAAC (R) & & \\
\hline
\end{tabular}

Direction of primers: $F$, forward; $R$, reverse. 
TABLE II. HLA-A and HLA-B Typing of Prostate and Breast Cancer Cell Lines

\begin{tabular}{lcc}
\hline Cell line & HLA-A $^{*}$ & HLA-B $^{*}$ \\
\hline LNCaP & 0101,0201 & 0801,3701 \\
PC-346C & 0101,0201 & 0801,4001 \\
PC-3 & 0101,2402 & 1801 \\
DU145 & 0301,3303 & 5001,5701 \\
BPH-1 & 0301,1101 & 1501,1801 \\
PNT2-C2 & 0101,3201 & 0702,5701 \\
MCF-7 & 0201 & 1801,4402 \\
T47D & 3301 & 1402 \\
\hline
\end{tabular}

many cases the full HLA genotypes of the cell lines are not known. We sequenced the HLA-A and HLA-B loci in four prostate cancer cell lines (LNCaP, PC-346C, PC3 , and DU145), two immortalized normal prostate epithelial cell lines (BPH-1 and PNT2-C2) and two breast cancer cell lines (MCF-7 and T47D). The results are presented in Table II. HLA-A*0201 is the most commonly targeted human MHC class I molecule in $\mathrm{T}$ cell-based immunotherapy. Of the cell lines sequenced herein, LNCaP, PC-346C, and MCF-7 have an HLA$\mathrm{A}^{*} 0201$ genotype.

\section{Cell Surface Expression of Immune Cell Interacting Molecules}

The expression level of MHC class I molecules (HLA-ABC) on the surface of target cells is crucial for CTL-mediated cell lysis. Therefore, we analyzed the expression level of HLA-ABC and HLA-A2 by flow cytometry on the panel of cell lines. The results are presented in Figure 1. We observed that the HLA-ABC expression varied substantially among cell lines with LNCaP and PC-346C displaying the lowest and PC-3 the highest HLA-ABC expression, Figure 1A. We also found that the HLA-ABC expression on LNCaP and PC-346C was not effected by IFN- $\gamma$ stimulation while PC-3, DU145, BPH-1, PNT2-C2, MCF-7, and T47D substantially upregulated HLA-ABC cell surface expression upon IFN- $\gamma$ stimulation. The HLA-A2 expression correlated well with the overall HLA-ABC expression on the HLA-A*0201 positive cell lines, Figure 1B.

The expression levels of costimulatory molecules and adhesion molecules are important for $\mathrm{T}$ cell activation and to some extent for CTL-mediated target cell lysis. We therefore analyzed the cell surface expression of CD40, CD80, CD86, and CD54 as well as HLA-DR by flow cytometry on the panel of cell lines. The results are presented in Table III. The expression of the costimulatory molecules CD40, CD80 and CD86

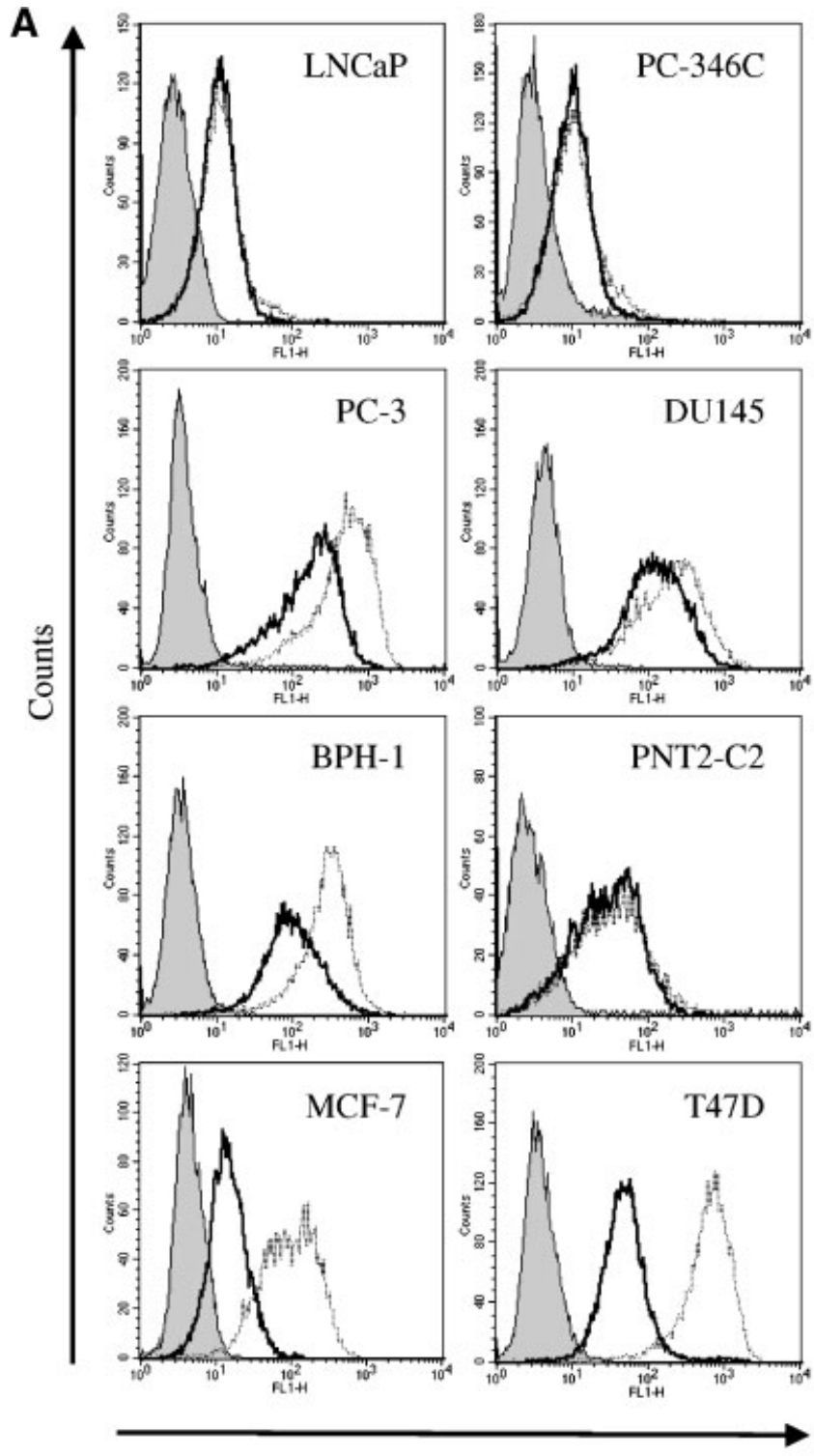

HLA-ABC

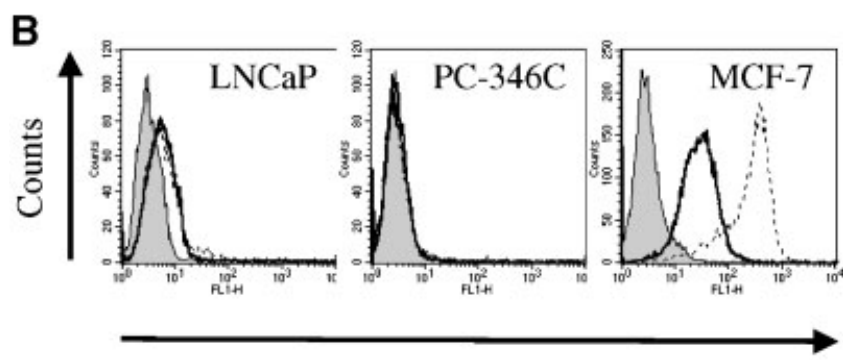

HLA-A2

Fig. I. HLA-ABC and HLA-A2 cell surface expression on prostate and breast cell lines. The cell lines were cultured in the presence (thin line) or absence (thick line) of I,000 IU/ml IFN- $\gamma$ for $72 \mathrm{hr}$. They were stained with an anti-HLA-ABC antibody (A) or an anti-HLA-A2 antibody (B) and analyzed by flow cy tometry. Filled curves represent isotype-matched background controls. 


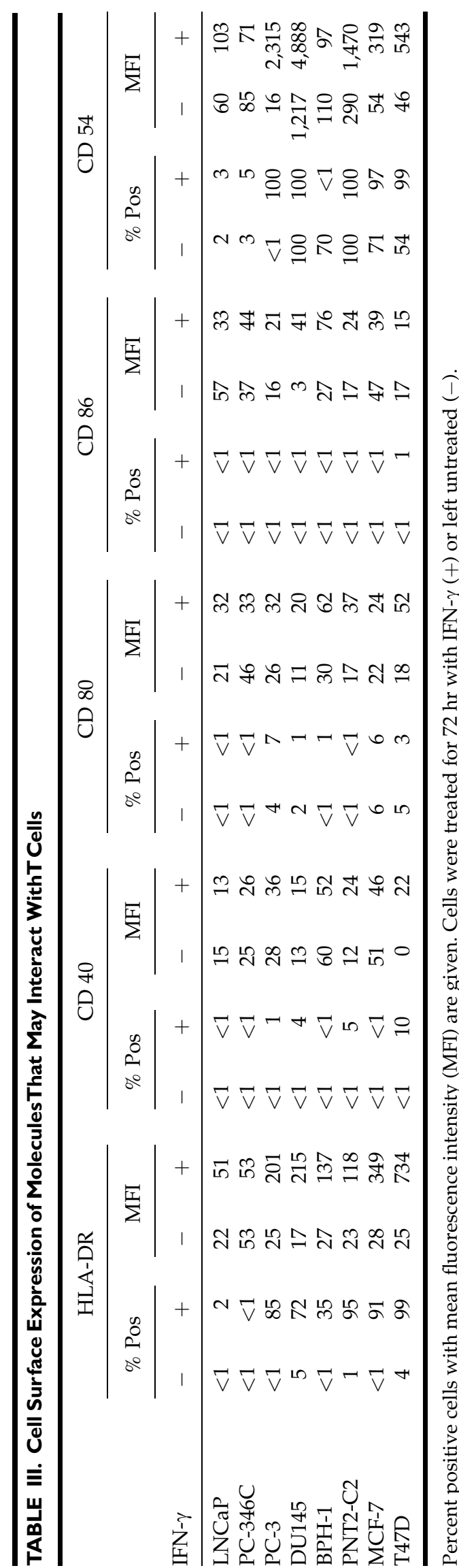

were non-existing or very low for all cell lines and IFN$\gamma$ treatment did not change this fact. The adhesion molecule CD54, on the other hand, was relatively highly expressed by all cell lines except for LNCaP and PC-346C. IFN- $\gamma$ treatment marginally increased the expression on CD54-positive cell lines except for BPH-1 where IFN- $\gamma$ treatment dramatically decreased CD54 expression. HLA-DR expression was relatively low but efficiently upregulated by IFN- $\gamma$ in all cell lines with the exception of LNCaP and PC-346C.

\section{Prostate Antigen mRNA Expression in Prostate and Breast Cancer Cell Lines}

For experimental immunotherapy it is important to have appropriate cell lines that express prostate antigens and present peptide epitopes derived from these antigens. We therefore set out to examine the expression of prostate-related genes in the panel of cell lines. RT-PCR analyses were preformed to determine mRNA expression of 25 genes reported to be specifically or preferentially expressed by normal and malignant prostate cells. The result of the screening is presented in Table IV. LNCaP expressed all examined genes while PC-346C expressed all genes except PSGR and NGEP. It is noteworthy that PSA expression is lost in the prostate cell lines PC-3, DU145, BPH-1, and PNT2-C2. The breast cancer cell line MCF-7 expressed 16 out of 25 genes while T47D expressed 8 out of 25 genes. STAMP1, Cten, AIbZIP, and PSDR1 were the only genes expressed by all examined cell lines.

\section{DISCUSSION}

In order to develop experimental immunotherapy for prostate cancer and breast cancer it is of outmost importance to have representative target cell lines that present relevant levels of peptides from TAAs on HLA class I molecules. Since HLA-A*0201 is the most common HLA class I molecule in humans, most studies describing the generation of $\mathrm{T}$ cells against prostate TAAs have focused on HLA-A*0201-restricted peptides [1]. Furthermore, most studies have used LNCaP as target cells despite the fact that LNCaP has an intracellular defect in the assembly of the MHC class I molecule [13-15]. In order to expand our knowledge of established prostate and breast cancer cell lines we sequenced the HLA-A and HLA-B loci of eight commonly used cell lines and analyzed their HLA$\mathrm{ABC}$ cell surface expression. We found that $\mathrm{LNCaP}$, PC-346C, and MCF-7 are HLA-A*0201 positive. However, the HLA-A2 protein expression level is low and among the three cell lines only MCF-7 upregulates HLA-A2 expression in response to IFN- $\gamma$ stimulation. This implies that even if $\mathrm{LNCaP}$ and PC-346C express prostate TAAs the presentation of relevant TAA 
TABLE IV. mRNA Expression of ProstateTissue Antigens in Prostate and Breast Cancer Cell Lines

\begin{tabular}{|c|c|c|c|c|c|c|c|c|}
\hline & LNCaP & PC-346C & PC-3 & DU145 & ВPH-1 & PNT2-C2 & MCF-7 & T47D \\
\hline PAP & + & + & - & - & - & - & - & - \\
\hline PSA & + & + & - & - & - & - & - & - \\
\hline hKLK4 & + & + & + & + & + & - & + & - \\
\hline PSP94 & + & + & - & - & - & - & + & - \\
\hline STAMP1 & + & + & + & + & + & + & + & + \\
\hline Cten & + & + & + & + & + & + & + & + \\
\hline hKLK2 & + & + & - & - & - & - & - & - \\
\hline TARP & + & + & - & - & - & - & + & - \\
\hline PDEF & + & - & + & - & - & - & + & + \\
\hline PSGR & + & + & - & - & - & - & + & - \\
\hline PART1 & + & + & - & + & + & + & + & + \\
\hline AIbZIP & + & + & + & + & + & + & + & + \\
\hline Trp-p8 & + & + & - & - & - & - & - & - \\
\hline POTE & + & + & - & - & - & + & + & - \\
\hline PMEPA1 & + & + & - & - & + & + & - & + \\
\hline PCTA1 & + & + & + & + & + & - & - & + \\
\hline Prostein & + & + & - & - & - & + & - & - \\
\hline PSMA & + & + & - & - & - & + & - & - \\
\hline PSDR1 & + & + & + & + & + & + & + & + \\
\hline TGM4 & + & + & + & - & + & + & - & - \\
\hline PSCA & + & + & + & + & + & + & + & - \\
\hline STEAP & + & + & + & + & + & + & + & - \\
\hline NGEP & + & - & - & - & - & - & + & - \\
\hline PrLZ & + & + & + & - & + & + & + & - \\
\hline NKX 3.1 & + & + & + & + & + & + & + & - \\
\hline$\beta$-actin & + & + & + & + & + & + & + & + \\
\hline
\end{tabular}

peptide epitopes may be too low for efficient CTL lysis. On the other hand, our data indicate that MCF-7 could, in many cases, be used as a complement to HLA$A^{*} 0201$ positive prostate cancer target cells for in vitro $T$ cell analysis. The drawback with MCF-7 is that it does not express all relevant prostate TAAs.

It has recently been shown that interaction between lymphocyte function-associated antigen-1 (LFA-1) on the CTL and CD54 (ICAM-1) on the target cell leads to a larger contact and a unique positioning of granules near the interface. This interaction directs the release of cytolytic granules to the surface of the antigen-expressing target cell and it mediates effective destruction of these cells by CTLs [16]. The fact that LNCaP and PC346C express low levels of CD54 may render them poor target cells for CTL lysis. On the other hand DU145, BPH-1, PNT2-C2, MCF-7, and T47D express high levels of CD54 that would improve their value as target cells.

Despite the identification of TAAs and their immunogenic peptide epitopes for use as cancer vaccines, there is a steady interest in developing allogeneic (MHC-disparate) cancer vaccines by using tumor cell lines [17,18]. For example, irradiated $\mathrm{LNCaP}$ and OnyCap23, a subclone of PNT2-C2, have been used as allogeneic prostate cancer vaccines [19]. However, the low expression levels of HLA-DR and costimulatory molecules (CD40, CD80, CD86) indicate that they are not optimal as antigen presenting cells for $\mathrm{T}$ cell activation. Therefore, to be efficient such therapeutic cancer vaccines would need to be targeted to professional antigen presenting cells such as dendritic cells. In addition, total RNA from LNCaP has recently been used as a source of prostate-related antigens in a phase I clinical trial [20]. Due to the wide expression of prostate-related antigens most prostate cancer cell lines analyzed in this article may be good sources of antigens for vaccination studies if combined with dendritic cells.

\section{ACKNOWLEDGMENTS}

This study was supported by the Swedish Cancer Society (Grant No. 4419-B05-06XBC) and the Swedish Research Council (Grant No. K2005-31X-15270-01A). ME is a recipient of the Göran Gustafsson's Award.

\section{REFERENCES}

1. Essand M. Gene therapy and immunotherapy of prostate cancer: Adenoviral-based strategies. Acta Oncol 2005;44(6):610-627. 
2. Sauter ER, Babb J, Daly M, Engstrom PF, Ehya H, Malick J, Diamandis E. Prostate-specific antigen production in the female breast: Association with progesterone. Cancer Epidemiol Biomarkers Prev 1998;7(4):315-320.

3. Sauter ER, Lininger J, Magklara A, Hewett JE, Diamandis EP. Association of kallikrein expression in nipple aspirate fluid with breast cancer risk. Int J Cancer 2004;108(4):588-591.

4. Horoszewicz JS, Leong SS, Kawinski E, Karr JP, Rosenthal H, Chu TM, Mirand EA, Murphy GP. LNCaP model of human prostatic carcinoma. Cancer Res 1983;43(4):1809-1818.

5. Kaighn ME, Narayan KS, Ohnuki Y, Lechner JF, Jones LW. Establishment and characterization of a human prostatic carcinoma cell line (PC-3). Invest Urol 1979;17(1):16-23.

6. Stone KR, Mickey DD, Wunderli H, Mickey GH, Paulson DF. Isolation of a human prostate carcinoma cell line (DU 145). Int J Cancer 1978;21(3):274-281.

7. Hayward SW, Dahiya R, Cunha GR, Bartek J, Deshpande $\mathrm{N}$, Narayan P. Establishment and characterization of an immortalized but non-transformed human prostate epithelial cell line: BPH-1. In Vitro Cell Dev Biol Anim 1995;31(1): 14-24.

8. van Weerden WM, de Ridder CM, Verdaasdonk CL, Romijn JC, van der Kwast TH, Schroder FH, van Steenbrugge GJ. Development of seven new human prostate tumor xenograft models and their histopathological characterization. Am J Pathol 1996; 149(3):1055-1062.

9. Lang SH, Stower M, Maitland NJ. In vitro modelling of epithelial and stromal interactions in non-malignant and malignant prostates. Br J Cancer 2000;82(4):990-997.

10. Keydar I, Chen L, Karby S, Weiss FR, Delarea J, Radu M, Chaitcik $\mathrm{S}$, Brenner HJ. Establishment and characterization of a cell line of human breast carcinoma origin. Eur J Cancer 1979;15(5):659670 .

11. Brooks SC, Locke ER, Soule HD. Estrogen receptor in a human cell line (MCF-7) from breast carcinoma. J Biol Chem 1973; 248(17):6251-6253.
12. Bengtsson M, Danielsson F, Jansson IE, Jidell E, Johansson U. Two new HLA $\mathrm{Cw}^{*}$ alleles, $\mathrm{Cw}^{*} 0105$ and $\mathrm{Cw}^{*} 1405$, detected by sequence based typing. Tissue Antigens 2002;59(3):226228.

13. Carlsson B, Totterman TH, Essand M. Generation of cytolytic T lymphocytes specific for the prostate and breast tissue antigen TARP. Prostate 2004;61(2):161-170.

14. Lu J, Celis E. Recognition of prostate tumor cells by cytotoxic $\mathrm{T}$ lymphocytes specific for prostate-specific membrane antigen. Cancer Res 2002;62(20):5807-5812.

15. Sanda MG, Restifo NP, Walsh JC, Kawakami Y, Nelson WG, Pardoll DM, Simons JW. Molecular characterization of defective antigen processing in human prostate cancer. J Natl Cancer Inst 1995;87(4):280-285.

16. Anikeeva N, Somersalo K, Sims TN, Thomas VK, Dustin ML, Sykulev Y. Distinct role of lymphocyte function-associated antigen-1 in mediating effective cytolytic activity by cytotoxic T lymphocytes. Proc Natl Acad Sci USA 2005;102(18):6437-6442.

17. Errington F, Bateman A, Kottke T, Thompson J, Harrington $K$, Merrick A, Hatfield P, Selby P, Vile R, Melcher A. Allogeneic tumor cells expressing fusogenic membrane glycoproteins as a platform for clinical cancer immunotherapy. Clin Cancer Res 2006;12(4):1333-1341.

18. Ward S, Casey D, Labarthe MC, Whelan M, Dalgleish A, Pandha $\mathrm{H}$, Todryk S. Immunotherapeutic potential of whole tumour cells. Cancer Immunol Immunother 2002;51(7):351-357.

19. Michael A, Ball G, Quatan N, Wushishi F, Russell N, Whelan J, Chakraborty P, Leader D, Whelan M, Pandha H. Delayed disease progression after allogeneic cell vaccination in hormoneresistant prostate cancer and correlation with immunologic variables. Clin Cancer Res 2005;11(12):4469-4478.

20. Mu LJ, Kyte JA, Kvalheim G, Aamdal S, Dueland S, Hauser M, Hammerstad H, Waehre H, Raabe N, Gaudernack G. Immunotherapy with allotumour mRNA-transfected dendritic cells in androgen-resistant prostate cancer patients. Br J Cancer 2005; 93(7):749-756. 